Article

\title{
eIF $2 \alpha$ Phosphorylation by GCN2 Is Induced in the Presence of Chitin and Plays an Important Role in Plant Defense against $B$. cinerea Infection
}

\author{
Marta Berrocal-Lobo ${ }^{1,2, *(\mathbb{D})}$, René Toribio ${ }^{1}$ (D) and M. Mar Castellano ${ }^{1, *(D)}$ \\ 1 Centro de Biotecnología y Genómica de Plantas, Universidad Politécnica de Madrid (UPM)—Instituto \\ Nacional de Investigación y Tecnología Agraria y Alimentaria (INIA), Campus de Montegancedo UPM, \\ 28223 Pozuelo de Alarcón, Madrid, Spain; toribio.francisco@upm.es \\ 2 Departamento de Sistemas y Recursos Naturales, E.T.S.I. Montes, Forestal y del Medio Natural, \\ Ciudad Universitaria s/n, 28040 Madrid, Spain \\ * Correspondence: m.berrocal@upm.es (M.B.-L.); castellano.mar@inia.es (M.M.C.); \\ Tel.: +34-910-679-181 (M.M.C.)
}

Received: 4 August 2020; Accepted: 1 October 2020; Published: 4 October 2020

\begin{abstract}
Translation plays an important role in plant adaptation to different abiotic and biotic stresses; however, the mechanisms involved in translational regulation during each specific response and their effect in translation are poorly understood in plants. In this work, we show that GCN2 promotes eIF2 $\alpha$ phosphorylation upon contact with Botrytis cinerea spores, and that this phosphorylation is required for the proper establishment of plant defense against the fungus. In fact, independent $g c n 2$ mutants display an enhanced susceptibility to $B$. cinerea infection, which is highlighted by an increased cell death and reduced expression of ethylene- and jasmonic-related genes in the gcn2 mutants. eIF $2 \alpha$ phosphorylation is not only triggered in the presence of the fungus, but interestingly, is also achieved in the sole presence of the microbe-associated molecular pattern (MAMP) chitin. Moreover, analysis of de novo protein synthesis by ${ }^{35 \mathrm{~S}} \mathrm{Met}-{ }^{35 \mathrm{~S}} \mathrm{Cys}$ incorporation indicates that chitin treatment promotes a global inhibition of translation. Taken together, these results suggest that eIF2 $\alpha$ phosphorylation by GCN2 is promoted in the presence of chitin and plays an important role in plant defense against $B$. cinerea infection.
\end{abstract}

Keywords: GCN2; eIF2 $\alpha$ phosphorylation; chitin; B. cinerea; necrotrophic fungi; translation regulation; defense-related genes

\section{Introduction}

Translation is highly regulated in plants during different developmental programs and in response to multiple stimuli [1-3]. However, despite the well-known relevance of regulation of translation in these eukaryotes, the mechanisms involved in translational control in the plant kingdom remain mainly unknown. This lack of knowledge is revealed by the observation that some important translational regulators in mammals and fungi are missing in plants and some others that seem to be conserved show a different level of specialization $[4,5]$.

In other eukaryotes, one of the best-known mechanisms for translational regulation is mediated by eukaryotic initiation factor 2 subunit $\alpha$ (eIF2 $\alpha)$ phosphorylation. This phosphorylation inhibits the conversion of eIF2-GDP to eIF2-GTP, blocking further cycles of translation initiation [6]. Among the different eIF2 $\alpha$ kinases described in eukaryotes, GCN2 (for general control non-derepressible 2) is the only eIF2 $\alpha$ kinase described in plants [2,4,5,7-9]. In other eukaryotes GCN2 has a prominent role in amino acid starvation response [10], and in accordance to this role in plants, AtGCN2 complements the $g c n 2$ yeast mutant strain in the presence of inhibitors of branched-chain and histidine amino acid 
biosynthesis [11]. In addition, AtGCN2 also phosphorylates eIF2 $\alpha$ during the treatment with herbicides that interfere with amino acid biosynthesis such as chlorsulfuron and glyphosate $[8,12]$. Furthermore, in vitro analysis using purified proteins revealed the ability of AtGCN2 to bind uncharged tRNA and to phosphorylate AteIF2 $\alpha$ [13]. All together, these results suggest that AtGCN2 directly phosphorylates AteIF2 $\alpha$ and highlight the conserved role of GCN2 as a sensor of amino acid availability in plants.

Plant GCN2 has also been involved in other specific developmental programs, abiotic stress responses, and defense [7,14,15]. In this latter sense, it has been described that GCN2-mediated eIF2 $\alpha$ phosphorylation is induced by wounding and by hormones involved in plant defense such as salicylic acid (SA), jasmonic acid (JA), methyl jasmonate (MeJA), and the ethylene (ET) precursor 1-aminocyclopropane-1-carboxylic acid (ACC) [7,14]. In addition, GCN2-mediated eIF2 $\alpha$-P is induced by the presence of the whitefly Bemisia tabaci in tobacco plants [14] and in response to bacterial infection by P. syringae pv. tomato (Pst) strains DC3000 and DC3118 in Arabidopsis [16]. Despite these pieces of evidence suggesting a role of GCN2 and eIF $2 \alpha$ phosphorylation in plant defense, it is still unclear whether eIF $2 \alpha$ phosphorylation can play a main role in other pathogen responses and which are the molecular players involved in the activation of the GCN2 pathway. This produces a gap in the knowledge on the possible effect of eIF $2 \alpha$ in the regulation of translation during plant immunity.

\section{Results}

\section{1. gen2 Mutants Show Impaired Defense Response against Botrytis cinerea}

In order to analyze if GCN2 plays a role during the plant defense response to B. cinerea, two independent Col-0 Arabidopsis gcn2 mutants, gcn2-2 (SALKseq_032196) and gcn2-3 (SALKseq_129334) were inoculated with B. cinerea. As shown in Figure 1A, compared to the wild-type plants (Col-0), both gcn2 mutants showed severer necrotic lesions and chlorosis symptoms upon infection with the fungus. In addition, they showed a higher level of cell death on the infected leaves (analyzed by Trypan blue staining). These symptoms were correlated with an increased ion leakage in the gcn 2 mutant lines (Figure 1B).

It is well-known that ET and JA pathways play a main role in plant defense against $B$. cinerea infection [17-19]; therefore, to determine if this higher susceptibility is also reflected at the molecular level, we carried out qRT-PCR analysis of ET and JA marker genes. Since both mutants showed a similar phenotype, we selected for these experiments one of the gcn 2 mutant lines. As shown in Figure 1C, compared to the wild-type plants, with the only exception of $P R 1$, the induction in the expression of the other five ET and JA marker genes was reduced in the gcn2-2 mutant upon B. cinerea contact. These results suggest that the defense transcriptional response to $B$. cinerea is not fully accomplished in gcn2 mutants.

Altogether, the results indicate that GCN2 is involved in plant immunity against the necrotrophic fungus $B$. cinerea. These results are in accordance with the reduced induction of ET- and JA-related mRNAs in the gcn 2 mutant analyzed by (Faus et al., 2015) and with the high production of MeJA and the lower accumulation of SA in the NtGCN2 overexpressing plants [14]. 
A

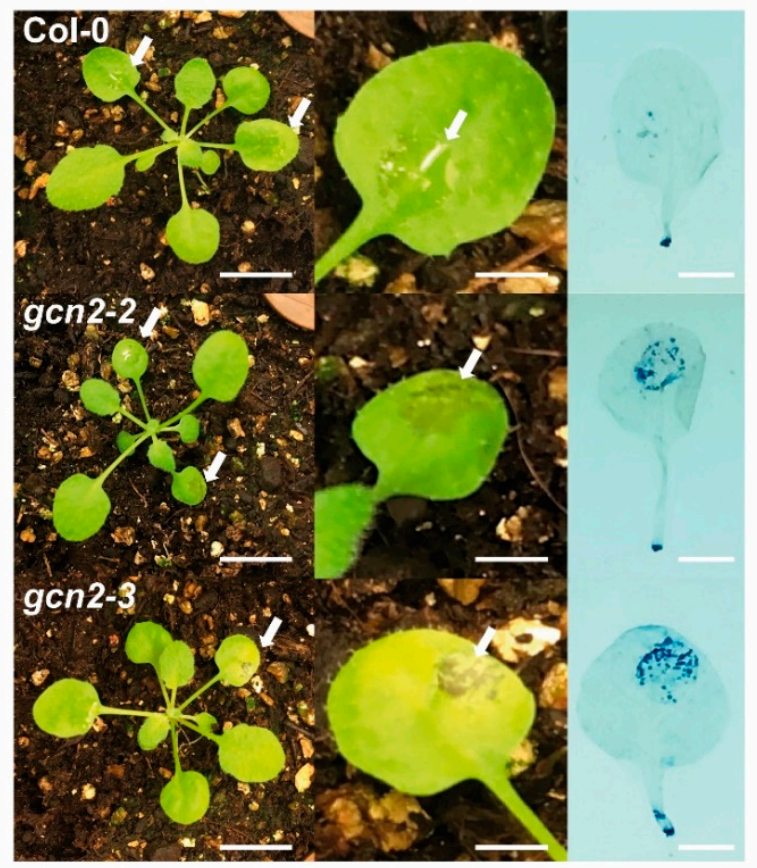

B
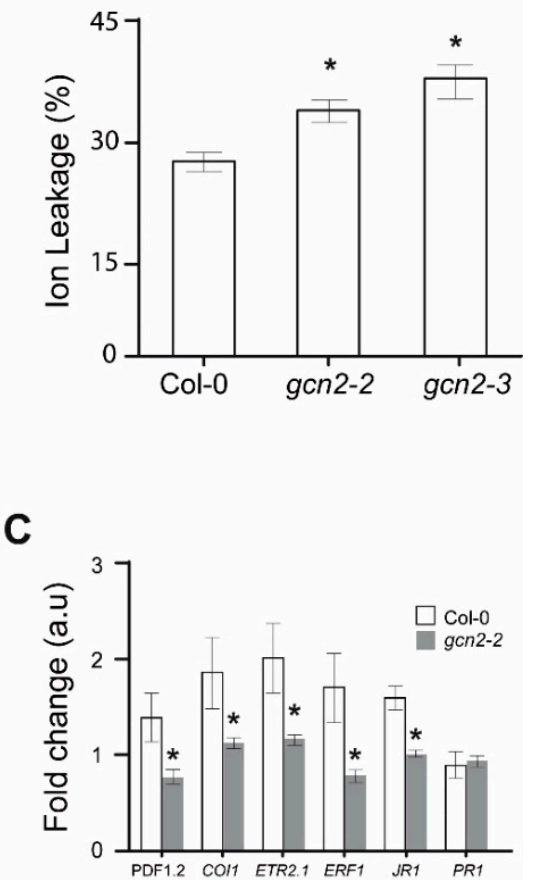

Figure 1. AtGCN2 participates in the defense response to Botrytis cinerea. (A) Representative disease symptoms in 3-week-old seedlings from Col-0 (WT) and gcn2-2 (SALKseq_032196) and gcn2-3 (SALKseq_129334) mutants at 3 dpi (right panel). For each experiment, two leaves from each plant were inoculated with $5 \mu \mathrm{L}$ of a Botrytis suspension $\left(5 \times 10^{6}\right.$ spores $\left./ \mathrm{mL}\right)$. Close-up views of a representative $B$. cinerea infected leaf from each genotype (middle panel). In these two panels, lesions are highlighted with white arrows. Representative cell death symptoms on leaves detected by Trypan blue staining (right panel). Scale bars correspond to $1 \mathrm{~cm}$ in the left panel and $0.25 \mathrm{~cm}$ in the middle and right panels. These experiments were repeated independently at least three times, obtaining similar results. (B) Analysis of ion leakage from infected leaves of the same lines at $3 \mathrm{dpi}$. The percentage of ion leakage upon Botrytis inoculation is related to the total conductivity of the leaves after membrane and wall breakdown. $n=6$ independent experiments including 10 leaves per genotype. Statistical differences $\left({ }^{*} p \leq 0.05\right)$ are highlighted by asterisks. (C) qRT-PCR analysis of AtPDF1.2, AtCOI1, AtETR2.1, AtERF1, AtJR1, and AtPR1 transcription levels at $1 \mathrm{dpi}$. Fold change expression is related to the expression of each gene in the corresponding genotype under control conditions (i.e., in the absence of fungus), which was arbitrarily assigned value 1 after normalization to $\beta$-ACTIN (internal normalization control). In this case, three biological replicates were analyzed. For (B and C), values are shown as mean \pm SEM. In both cases, statistically significant differences $\left({ }^{*} p<0.01\right)$ were calculated using one-way ANOVA test.

\section{2. eIF2 $\alpha$ Is Phosphorylated upon Plant Contact with B. cinerea Spores and This Phosphorylation Is Dependent on GCN2}

Since the results described above suggest that GCN2 is involved in plant defense against $B$. cinerea, and GCN2 has been described as an eIF2 $\alpha$ kinase in plants [11], we decided to study if GCN2 promotes eIF $2 \alpha$ phosphorylation during incubation with the fungus. To do so, seedlings from Col- 0 wild-type genotype and the gcn2-3 mutant were incubated with $B$. cinerea spores. Subsequently, the incubated plants were collected, and eIF2 $\alpha$ phosphorylation was detected by Western blot using an anti-phospho-eIF2 $\alpha$ antibody. As shown in Figure 2A, compared to the mock treated plants, eIF2 $\alpha$ phosphorylation was prompted in plants upon contact with $B$. cinerea. This phosphorylation was dependent on GCN2, since no phosphorylation was observed in the gcn2 mutant. 


\subsection{Chitin Induces GCN2-Dependent eIF2 $\alpha$ Phosphorylation}

One of the first processes that takes place on the plant surface during the establishment of the defense is the recognition of the MAMP chitin of the fungus [20]; therefore, in order to analyze if eIF2 $\alpha$ phosphorylation is triggered upon fungus recognition, we decided to study eIF $2 \alpha$ phosphorylation in response to chitin. For this, Col-0 Arabidopsis seedlings were incubated in the presence or absence of chitin and eIF2 $\alpha$ phosphorylation was monitored by Western blot (Figure 2B, left part). In parallel, we also analyzed chitin-triggered phosphorylation in the gcn2-2 and gcn2-3 mutants (Figure 2B, middle part). As positive controls of eIF2 $\alpha$ phosphorylation, we included a treatment with UV light, a well-known agent that promotes eIF2 $\alpha$ phosphorylation in plants [7] (Figure 2B, right part). As shown in Figure 2B, as it was also the case of the UV treatment, chitin-promoted eIF2 $\alpha$-phosphorylation and this phosphorylation was fully abolished in the gcn2-2 and gcn2-3 mutants. All these data highly suggest that chitin prompts eIF $2 \alpha$ phosphorylation in plants and that this phosphorylation is dependent on GCN2.
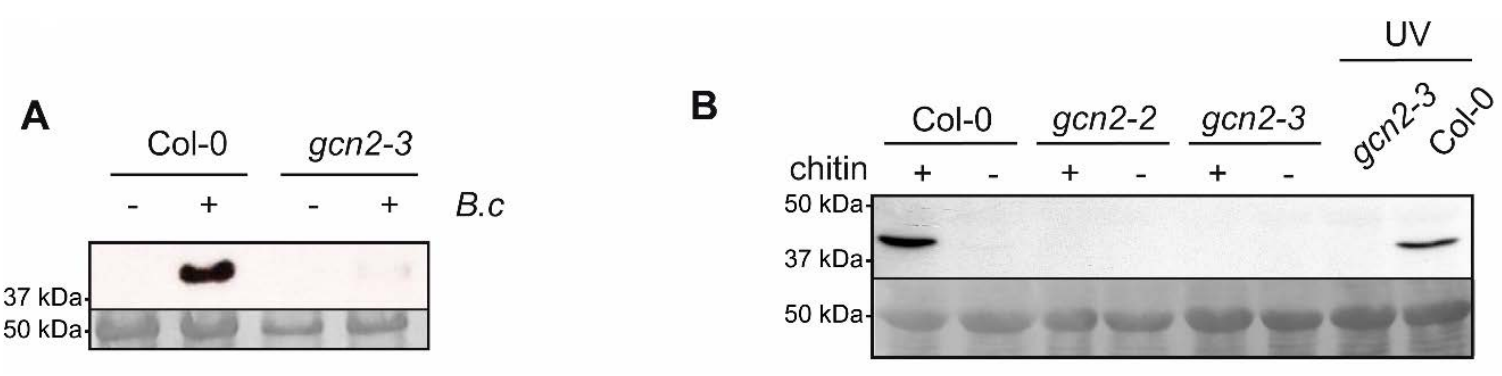

Figure 2. GCN2-dependent eIF2 $\alpha$ phosphorylation is induced upon contact with $B$. cinerea spores and by chitin. (A) Analysis of eIF2 $\alpha$-P of wild-type (Col-0) and gcn2-3 (SALKseq_129334) seedlings mock inoculated (-) or incubated with a suspension of $5 \times 10^{6}$ spores $/ \mathrm{mL}$ of Botrytis cinerea (B.c) (+) for $60 \mathrm{~min}$. (B) Analysis of eIF2 $\alpha$ phosphorylation of wild-type (Col-0), gcn2-2 (SALKseq_032196) and gcn2-3 (SALKseq_129334) seedlings incubated in the presence of $400 \mathrm{mg} / \mathrm{L}$ chitin (+) or in the absence of chitin (-) for $1 \mathrm{~h}$. As control, Col-0 seedlings were subjected to an UV treatment known to promote eIF $2 \alpha$ phosphorylation. In all cases, 7-day-old seedlings were used for the experiments and eIF2 $\alpha$ phosphorylation was analyzed by Western blot (upper panels). As loading control of each experiment, representative bands from the Coomassie staining of the original membrane are provided (lower panels). All the experiments were independently repeated at least three times obtaining similar results.

To provide further evidence on the possible conservation of this phosphorylation in a different ecotype, we decided to analyze if chitin also promotes eIF $2 \alpha$ phosphorylation in the Ler genotype, and for this, we took advantage of the previously described gcn2 mutant GT8359 [8]. As observed in Supplementary Figure S1, we also observed a GCN2-dependent phosphorylation by chitin in this different background, which further reinforces that this mechanism is conserved in different Arabidopsis ecotypes.

\subsection{Chitin Promotes a Global Reduction of Translation}

It is well-known that eIF $2 \alpha$ phosphorylation leads to a global inhibition in protein translation in mammals and yeast [10]. However, despite multiple pieces of evidence indicating that eIF2 $\alpha$ phosphorylation is induced in plants in response to different stresses, including upon SA, JA, and ACC treatments [7], whether this phosphorylation is associated to global changes in translation has remain elusive, except in the case of treatments with chlorsulfuron and 8-azaadeninine (AZA), which course with an accumulation of uncharged tRNAs.

Thus, in order to investigate whether chitin perception leads to a global effect on plant translation, Arabidopsis seedlings were treated with different concentrations of chitin, and subsequently, the seedlings were labeled with ${ }^{35}$ S-Methionine ${ }^{35}$ S-Cysteine. After labeling, equal amounts of total 
protein were loaded in a gel and run. The gel was subjected to Coomassie staining (Figure 3A, lower panel, provided as loading control) and ${ }^{35} \mathrm{~S}$-Methionine ${ }^{35} \mathrm{~S}$-Cysteine incorporation into the de novo synthesized proteins was analyzed by autoradiography (Figure 3A, upper panel). As positive control, we also monitored protein synthesis in seedlings subjected to heat stress, a treatment that promotes a drastic inhibition of protein translation [1]. As shown in Figure 3A, upper panel, chitin induced a reduction in the global protein synthesis that was enhanced at higher concentrations of chitin. In the case of the treatments carried out at $400 \mathrm{mg} / \mathrm{L}$ chitin, the reduction in de novo protein synthesis was quantified (Figure 3B). This analysis indicates that chitin promotes a significant reduction in the de novo protein synthesis.

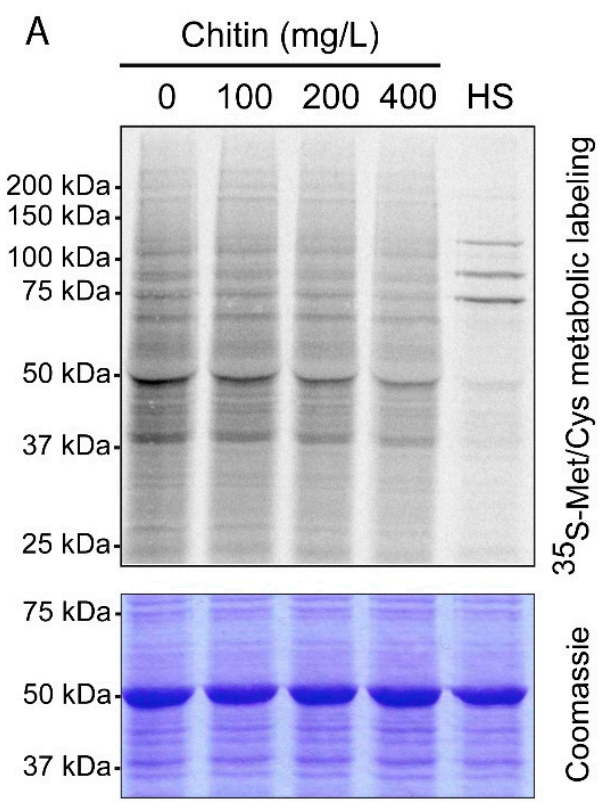

B

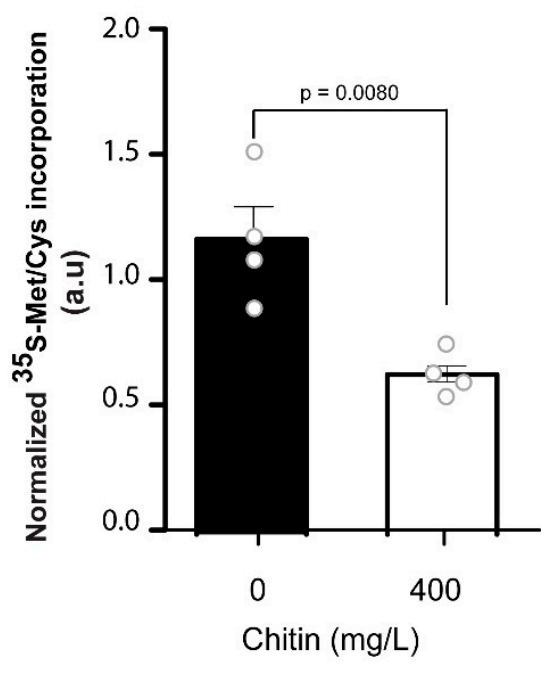

Figure 3. Chitin triggers a global reduction in protein synthesis. (A) Metabolic labeling of de novo synthesized proteins. Arabidopsis 7-day-old seedlings were incubated with ${ }^{35}$ S-Methionine ${ }^{35}$ S-Cysteine in the absence of chitin (-) or in the presence of different chitin concentrations (upper panel). As positive control of global inhibition of protein synthesis, seedlings were treated at $38^{\circ} \mathrm{C}$ for $30 \mathrm{~min}$ (HS). The Coomassie staining of the gel is provided as loading control (lower panel). This experiment was repeated at least three times obtaining similar results. (B) Normalized quantification of the ${ }^{35} \mathrm{~S}-$ Methionine ${ }^{35} \mathrm{~S}$-Cysteine incorporation into proteins (related to the total amount of loaded protein per lane) in the absence (-) or in the presence of chitin $(400 \mathrm{mg} / \mathrm{L})$. The bars show the mean \pm SEM of $n=4$ independent experiments. Statistically significant differences $(p<0.008)$ were calculated by $t$-student test.

In order to corroborate the chitin-induced reduction in protein synthesis by a different technique, we also carried out polysome profile analyses. To do so, extracts from Arabidopsis seedlings incubated in the absence or presence of $400 \mathrm{mg} / \mathrm{L}$ chitin were subjected to an ultracentrifugation in a $10-50 \%$ sucrose gradient. This technique allows the separation of mRNAs by density according to the number of ribosomes they are associated with. In such a way, those RNAs associated to multiple ribosomes, and so active in translation, migrate to the heavier fractions of the gradient that are called polysomal fractions. As shown in Supplementary Figure S2, compared to the control conditions, a modest decrease in polysomal fractions was observed in the chitin-treated plants, providing an alternative indication of the global inhibition of translation upon chitin treatments. All these results suggest that chitin triggers a GCN2-dependent eIF2 $\alpha$-phosphorylation in plants and a global reduction in protein synthesis. 


\section{Discussion}

Translation has recently emerged as a main mechanism to regulate gene expression during plant development and, especially, under environmental challenges [3-5,9]. Translation consumes a substantial amount of cellular energy, and so, upon environmental challenges, translation should be adjusted to reduce the consumption of energy while allowing the translation of key proteins involved in plant adaptation to stress. This is achieved by the establishment of a global translation inhibition and the selective synthesis of key stress regulators. Despite the output of this regulation being well-established, the knowledge about how this translational regulation is achieved, and the targets of this regulation is extremely scarce in the plant kingdom compared to other eukaryotes $[2,4,5,9]$.

Constitutive plant immunity involves the activation of basal defense after recognition of pathogen/microbe-associated molecular patterns (PAMPs/MAMPs) by pattern-recognition receptors (PRRs) [21]. MAMPs embrace a wide diversity of conserved molecules across a wide range of microbes, being pathogenic or not. Their recognition contributes to the plant basal resistance through the activation of pathogen-triggered immunity (PTI), during compatible and non-compatible interactions. An example of PAMPs is chitin (a $\beta$-linked sugar polymer that is present in the structures of fungal spores, yeast cell walls, insect exoskeletons, and shells of arthropods and invertebrates) [22].

Until now, different studies demonstrated that GCN2 is involved in eIF2 $\alpha$ phosphorylation in response to other pathogens and to different phytohormones associated to plant immunity $[7,14,15]$. In this study, we report that GCN2 is also involved in plant defense against B. cinerea infection. This conclusion is supported by the higher susceptibility to this fungus of the gcn 2 mutants, which is accompanied by a reduced expression of genes involved in different steps of the ET- and JA-signaling pathways. Furthermore, this is the first time that the role of a MAMP (and specifically chitin) in eIF2 $\alpha$ phosphorylation and in the regulation of global translation in plants has been addressed. Although the effect of chitin in protein translation could be observed at lower concentration of chitin (100 and $200 \mathrm{mg} / \mathrm{L}$, Figure 3), the strongest effect in inhibition of protein synthesis is achieved at $400 \mathrm{mg} / \mathrm{L}$ chitin, which prompted us to select this concentration for our experiments. This concentration, although higher than the used in normal basis $(100 \mathrm{mg} / \mathrm{L})$, is in the range of the chitin concentrations proven to produce effects related to defense in plants (such as callose deposition) [23]. Furthermore, the phosphorylation observed in Ler background in response to 100 and $200 \mathrm{mg} / \mathrm{L}$ chitin strongly suggests that this phosphorylation could be achieved in a lower range of chitin concentrations (Figure S1).

As observed, the reduction in global translation induced by chitin is moderate, especially when compared with the extreme global translation induced by heat stress. In this sense, it has to be considered that translation constitutes a fundamental step in gene expression, and therefore, changes in global translation should be finely controlled to allow a precise adaptation response to the environment. Accordingly, it seems logical that the effect (in quantitative terms) on translatome remodeling depends on the severity of the stress and on the damage induced by it. Our translation experiments were carried out in response to chitin treatments. Under such conditions, it is not expected that plants are subjected to a severe stress (as compared to heat), but to a priming process that may be achieved with more moderate changes in global translatome. Whether eIF $2 \alpha$ phosphorylation is the sole trigger of this translational inhibition seems improbable. It should be considered that there are multiple mechanisms of translation inhibition for assuring proper gene expression and energy saving under stress conditions, and eIF $2 \alpha$ phosphorylation is just one of them. In this sense, it is possible that other mechanisms of translation regulation could be involved and show different degree of influence in this translational control (Figure 4). 


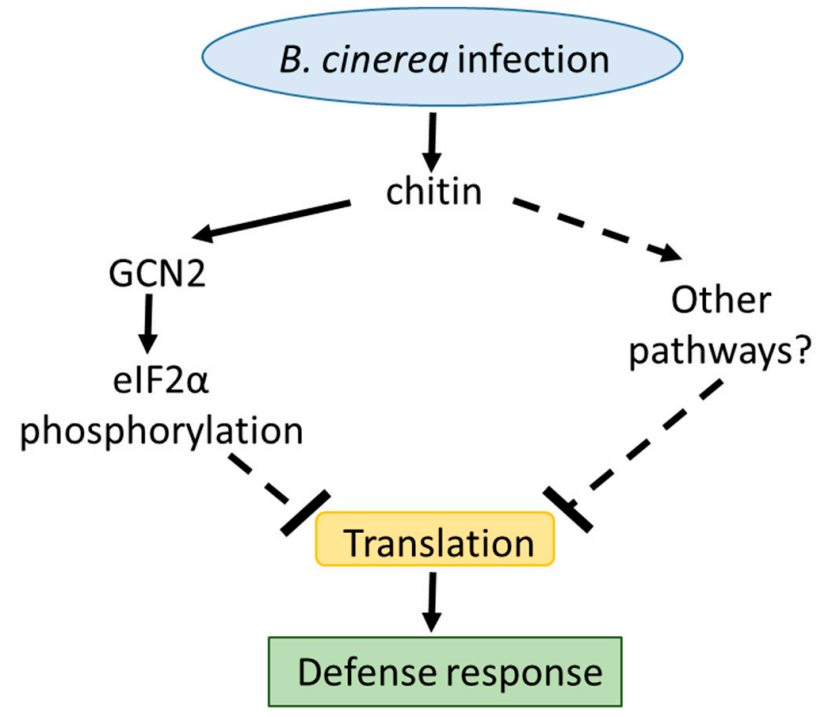

Figure 4. Proposed model for translation inhibition in response to chitin. Our results strongly suggest that chitin perception induces translation inhibition in plants. Chitin promotes, via activation of GCN2, the phosphorylation of eIF2 $\alpha$. This mechanism may contribute, most probably in concert with others, to translation inhibition in response to this signaling molecule. This inhibition of translation, most probably along with the selective translation of proteins involved in coping with the stress, may contribute to the proper activation of plant defense. The main role of regulation of translation in plant immunity has also been highlighted by other authors such as $[24,25]$. Solid and dashed lines represent proven and unknown connections among the processes, respectively.

\section{Materials and Methods}

\subsection{Plant Material and Growth Conditions}

For in vitro growth, Arabidopsis seeds were surface sterilized using 0.05\% (v/v) Tween-20 and 80\% $(v / v)$ ethanol for $5 \mathrm{~min}$ and stratified at $4{ }^{\circ} \mathrm{C}$ in darkness for $48 \mathrm{~h}$. The sterilized seeds were germinated on Murashige and Skoog (MS) medium (Duchefa, Haarlem, The Netherlands) supplemented with 1\% $(w / v)$ sucrose and $9 \mathrm{~g} / \mathrm{L}$ of plant agar. The plates were maintained in vertical position under long-day photoperiodic conditions (16/8 h light/dark schedule) at $22^{\circ} \mathrm{C}$ constant temperature. For growth in soil, Arabidopsis plants were grown in a mixture of soil-vermiculite (3:1) under long-day photoperiodic conditions at $22{ }^{\circ} \mathrm{C}$ day $/ 19{ }^{\circ} \mathrm{C}$ night temperature, $65 \%$ relative humidity, and $120 \mu \mathrm{Em}^{-2} \mathrm{~s}^{-1}$ of light intensity.

The gcn2 mutant lines GT8359 (Landsberg erecta background), SALKseq_032196 and SALKseq_129334 (these two latter lines in Col-0 background) were obtained from the NASC (Nottingham Arabidopsis Stock Centre, available online http://arabidopsis.info/BasicForm). When obtained, the lines SALKseq_032196 and SALKseq_129334 contained, in addition to a T-DNA insertion in the GCN2 gene, other T-DNA insertions in other parts of the genome, respectively. These lines were crossed to Col-0 and segregated until no amplification of the T-DNA sequences flanking the additional insertions were obtained in the gcn 2 homozygous mutants. During the course of this work, the lines SALKseq_032196 and SALKseq_129334 were described and named as gcn2-2 and $\operatorname{gcn} 2-3$ [26,27], respectively. This nomenclature has been maintained in the text and figures of the article.

\subsection{Phytopathogens, Storage, and Growth Conditions}

The fungal pathogen Botrytis cinerea [28] was grown on potato dextrose agar medium (PDA) at $28^{\circ} \mathrm{C}$ for 8 days, as previously described in [17]. Spores were collected in sterile water, filtered, quantified with a Neubauer chamber, and stored in $20 \%$ glycerol at $-80{ }^{\circ} \mathrm{C}$ until use. 


\subsection{Pathogen Inoculation and Phenotypic Assessment}

Three-week-old Abidopsis plants were used for pathogen inoculation experiments. For phenotypic assessment plant leaf surfaces were inoculated with $5 \mu \mathrm{L}$ of $B$. cinerea fungal inoculum $\left(5 \times 10^{6} \mathrm{spores} / \mathrm{mL}\right)$ in potato dextrose broth medium (PDB). Inoculated plants were placed in a growth chamber allowing fungal growth until harvesting. Cell death was determined by Trypan blue staining after 3 days post inoculation (dpi), as described in [29]. In the case of ion leakage analysis, inoculated leaves were harvested $3 \mathrm{dpi}$ and incubated in water for $4 \mathrm{~h}$, when the initial conductivity of the solution was measured. This value was normalized to the total ion content of the analyzed leaves (obtained by the analysis of the total conductivity of the solution after membrane and wall breakdown by incubation at $-80{ }^{\circ} \mathrm{C}$ ). Six independent replicates were assayed, each of them included at least 10 leaves per genotype. Significant differences were calculated using ANOVA test and the Statgraphics ${ }^{\circledR}$ program (version Centurion XV), Statgraphics Technologies, Inc., The Plains, Virginia, USA.

\subsection{Gene Expression Analysis by Quantitative Real-Time PCR (qRT-PCR)}

Four leaves from 4 different inoculated plants per genotype were harvested at $1 \mathrm{dpi}$. RNA isolation was carried out using the TRIzol reagent (Thermo Fisher). qRT-PCR analysis was performed as described in [30], using $1 \mu \mathrm{g}$ RNA for cDNA synthesis and $\beta$-ACTIN (At3g18780) for internal normalization. Fold change expression was related to the expression of the gene in the corresponding genotype under control conditions (i.e., in the absence of fungus), which was arbitrarily assigned value 1 after normalization to $\beta$-actin. Three biological replicates, each of them including three technical replicates, were analyzed. Primer sequences are listed in Supplementary Table S1. Statistically significant differences were calculated using paired Student's t-test using the analysis tool Statgraphics ${ }^{\circledR}$ (version Centurion XV program), Statgraphics Technologies, Inc., The Plains, Virginia, USA.

\section{5. eIF2 $\alpha$ Phosphorylation Analysis}

For the analysis of eIF $2 \alpha$ phosphorylation in response to Botrytis cinerea, 10 seedlings were transferred to tubes containing $B$. cinerea spores in liquid MS medium. Controls were incubated in a similar solution in absence of the fungus. For the analysis of eIF $2 \alpha$ phosphorylation in response to chitin, five to ten 7-day-old Arabidopsis seedlings (grown in vertical position) were transferred to a 24-well-plate and incubated for $1 \mathrm{~h}$ at $20^{\circ} \mathrm{C}$ in $1.2 \mathrm{ml}$ of either MiliQ water (control conditions) or MiliQ water supplemented with $400 \mathrm{mg} / \mathrm{L}$ chitin from shrimp shells (SIGMA \# C9752), previously autoclaved for $20 \mathrm{~min}$ to enhance solubilization. As controls of eIF2 $\alpha$ phosphorylation, the seedlings were exposed to UV-C radiation $[7,8])$. After the treatments, seedlings were quickly frozen in liquid nitrogen, ground to powder, and solubilized in Laemmli sample buffer. An amount of $20 \mu \mathrm{g}$ of protein extracts were loaded into $12 \%$ SDS-PAGE gels, blotted to nitrocellulose membranes, and analyzed with a specific phosphoantibody that recognizes the Ser51 eIF2 $\alpha$-phosphorylated form (Ref:9721, Cell Signaling, $1 / 1000$ dilution).

\subsection{Metabolic Labeling of Newly Synthesized Proteins}

Labeling of newly synthesized proteins was carried out as described in [1] with the following modifications: 7-day-old seedlings subjected to a $38^{\circ} \mathrm{C}$ heat stress treatment for $45 \mathrm{~min}$ (HS control) or treated with different chitin concentrations were supplemented with $50 \mu \mathrm{Ci} / \mathrm{mL}$ ${ }^{35 S}$ Methionine $/{ }^{35 S}$ Cysteine (EasyTagTM EXPRESS35S Protein Labeling Mix, Perkin Elmer, Waltham, MA, USA) for $15 \mathrm{~min}$. Seedlings were washed three times with water, quickly frozen in liquid nitrogen, ground to powder, and solubilized in 1x Laemmli sample buffer. Equivalent amounts of plant extracts were subjected to $10 \%$ SDS-PAGE electrophoresis, and ${ }^{35}$ S-labeled proteins were detected by autoradiography using Fuji BAS-SR type image plates and a Typhoon scanner. Coomassie staining of the gels was carried out and used as protein loading controls. Intensities of radiolabeled and Coomassie lanes from the gels were estimated using ImageJ software [31]. In each case, the intensity of 
the label of ${ }^{35 S}$ Met ${ }^{35 S}$ Cys was normalized to the intensity of each loading control. Four independent biological replicates were used for quantification. Statistically significant differences were calculated by Student's $t$-test.

\subsection{Polysome Profiling}

Seven-day-old seedlings treated in the absence or presence of $400 \mathrm{mg} / \mathrm{L}$ chitin were harvested and immediately frozen in liquid nitrogen. An amount of $700 \mathrm{mg}$ of frozen tissue were pulverized in liquid nitrogen with a mortar and pestle. Polysome analysis was carried out as described in [32].

Supplementary Materials: Supplementary materials can be found at http://www.mdpi.com/1422-0067/21/19/7335/ s1. Supplementary Table S1. Primers used in this study for PCR and real time qRT-PCR analysis. Supplementary Figure S1. Analysis of eIF2 $\alpha$ phosphorylation of wild-type (Ler) and gcn2 (GT8359) seedlings incubated in the absence of chitin (-) or in the presence of different concentrations of chitin for $1 \mathrm{~h}$. Seven-day-old seedlings were used for the experiments, and eIF $2 \alpha$ phosphorylation was analyzed by Western blot (upper panel). As loading control, representative bands from the Coomassie staining of the original membrane are provided. These experiments were repeated at least three times with similar results. Supplementary Figure S2. Polysome profiles of 7-day-old seedlings from Col-0 incubated for $1 \mathrm{~h}$ in the absence of chitin or in the presence of $400 \mathrm{mg} / \mathrm{L}$ chitin. The experiment was repeated three times.

Author Contributions: M.B.-L. characterized the $g c n 2$ mutants and designed and conducted the experiments related to the analysis of the B. cinerea response and eIF $2 \alpha$ phosphorylation. R.T., M.B.-L. and M.M.C. designed and M.B.-L. and R.T. carried out the experiments related to translation regulation in the presence of chitin. For correspondence about $g c n 2$ mutants, analysis of the B. cinerea response, and eIF $2 \alpha$ phosphorylatin, please contact M.B.-L. For correspondence about translational regulation, please contact M.M.C. All authors have read and agreed to the published version of the manuscript.

Funding: This research has received funding from the grants: S2013-ABI2734 from CAM, RTI2018-095946-B100 from MICIU and "Severo Ochoa Programme for Centres of Excellence in R\&D" from the Agencia Estatal de Investigación of Spain (grant SEV-2016-0672 (2017-2021) to the CBGP). In the frame of this latter program R. T. was supported with a postdoctoral contract.

Acknowledgments: We thank Plant Response and Antonio Molina (Centro de Biología y Genómica de Plantas) for kindly providing the B. cinerea strain. We also acknowledge Pablo González-Melendi (Centro de Biología y Genómica de Plantas) for technical support on microscopy and Alfonso Muñoz (Universidad de Córdoba) for his helpful comments on the manuscript.

Conflicts of Interest: The authors declare no conflict of interest. The funders had no role in the design of the study; in the collection, analyses, or interpretation of data; in the writing of the manuscript, or in the decision to publish the results.

\section{Abbreviations}

$\begin{array}{ll}\text { GCN2 } & \text { general control non-derepressible } 2 \\ \text { eIF2 } \alpha & \text { eukaryotic initiation factor } 2 \text { subunit } \alpha \\ \text { SA } & \text { salicylic acid } \\ \text { JA } & \text { jasmonic acid } \\ \text { MeJA } & \text { methyl jasmonate } \\ \text { ET } & \text { ethylene } \\ \text { ACC } & \text { 1-aminocyclopropane-1-carboxylic acid }\end{array}$

\section{References}

1. Yanguez, E.; Castro-Sanz, A.B.; Fernandez-Bautista, N.; Oliveros, J.C.; Castellano, M.M. Analysis of genome-wide changes in the translatome of Arabidopsis seedlings subjected to heat stress. PLoS ONE 2013, 8, e71425. [CrossRef]

2. Echevarria-Zomeno, S.; Yanguez, E.; Fernandez-Bautista, N.; Castro-Sanz, A.B.; Ferrando, A.; Castellano, M.M. Regulation of Translation Initiation under Biotic and Abiotic Stresses. Int. J. Mol. Sci. 2013, 14, 4670-4683. [CrossRef] [PubMed]

3. Merchante, C.; Stepanova, A.N.; Alonso, J.M. Translation regulation in plants: An interesting past, an exciting present and a promising future. Plant. J. 2017, 90, 628-653. [CrossRef] [PubMed] 
4. Sesma, A.; Castresana, C.; Castellano, M.M. Regulation of Translation by TOR, eIF4E and eIF2alpha in Plants: Current Knowledge, Challenges and Future Perspectives. Front. Plant. Sci. 2017, 8, 644. [CrossRef] [PubMed]

5. Munoz, A.; Castellano, M.M. Regulation of Translation Initiation under Abiotic Stress Conditions in Plants: Is It a Conserved or Not so Conserved Process among Eukaryotes? Comp. Funct. Genomics. 2012, 2012, 406357. [CrossRef] [PubMed]

6. Wek, R.C.; Jiang, H.Y.; Anthony, T.G. Coping with stress: eIF2 kinases and translational control. Biochem Soc. Trans. 2006, 34, 7-11. [CrossRef] [PubMed]

7. Lageix, S.; Lanet, E.; Pouch-Pelissier, M.N.; Espagnol, M.C.; Robaglia, C.; Deragon, J.M.; Pelissier, T. Arabidopsis eIF2alpha kinase GCN2 is essential for growth in stress conditions and is activated by wounding. BMC Plant. Biol. 2008, 8, 134. [CrossRef] [PubMed]

8. Zhang, Y.; Wang, Y.; Kanyuka, K.; Parry, M.A.; Powers, S.J.; Halford, N.G. GCN2-dependent phosphorylation of eukaryotic translation initiation factor-2alpha in Arabidopsis. J. Exp. Bot. 2008, 59, 3131-3141. [CrossRef] [PubMed]

9. Toribio, R.; Muñoz, A.; Castro-Sanz, A.B.; Ferrando, A.; Berrocal-Lobo, M.; Castellano, M.M. Evolutionary aspects of translation regulation during abiotic stress and development in plants. In Evolution of the Protein Synthesis Machinery and Its Regulation; Hernández, G., Jagus, R., Eds.; Springer: Heidelberg, Germany, 2016.

10. Hinnebusch, A.G. Translational regulation of GCN4 and the general amino acid control of yeast. Annu Rev. Microbiol. 2005, 59, 407-450. [CrossRef] [PubMed]

11. Zhang, Y.; Dickinson, J.R.; Paul, M.J.; Halford, N.G. Molecular cloning of an arabidopsis homologue of GCN2, a protein kinase involved in co-ordinated response to amino acid starvation. Planta 2003, 217, 668-675. [CrossRef]

12. Faus, I.; Zabalza, A.; Santiago, J.; Nebauer, S.G.; Royuela, M.; Serrano, R.; Gadea, J. Protein kinase GCN2 mediates responses to glyphosate in Arabidopsis. BMC Plant. Biol. 2015, 15, 14. [CrossRef] [PubMed]

13. Li, M.W.; AuYeung, W.K.; Lam, H.M. The GCN2 homologue in Arabidopsis thaliana interacts with uncharged tRNA and uses Arabidopsis eIF2alpha molecules as direct substrates. Plant. Biol. (Stuttg.) 2013, 15, 13-18. [CrossRef] [PubMed]

14. Li, N.; Zhang, S.J.; Zhao, Q.; Long, Y.; Guo, H.; Jia, H.F.; Yang, Y.X.; Zhang, H.Y.; Ye, X.F.; Zhang, S.T. Overexpression of Tobacco GCN2 Stimulates Multiple Physiological Changes Associated with Stress Tolerance. Front. Plant. Sci. 2018, 9, 725. [CrossRef] [PubMed]

15. Liu, X.; Merchant, A.; Rockett, K.S.; McCormack, M.; Pajerowska-Mukhtar, K.M. Characterization of Arabidopsis thaliana GCN2 kinase roles in seed germination and plant development. Plant. Signal. Behav. 2015, 10, e992264. [CrossRef] [PubMed]

16. Liu, X.; Afrin, T.; Pajerowska-Mukhtar, K.M. Arabidopsis GCN2 kinase contributes to ABA homeostasis and stomatal immunity. Commun. Biol. 2019, 2, 302. [CrossRef]

17. Berrocal-Lobo, M.; Molina, A.; Solano, R. Constitutive expression of ETHYLENE-RESPONSE-FACTOR1 in Arabidopsis confers resistance to several necrotrophic fungi. Plant. J. 2002, 29, 23-32. [CrossRef]

18. AbuQamar, S.; Moustafa, K.; Tran, L.S. Mechanisms and strategies of plant defense against Botrytis cinerea. Crit. Rev. Biotechnol. 2017, 37, 262-274. [CrossRef]

19. AbuQamar, S.; Chen, X.; Dhawan, R.; Bluhm, B.; Salmeron, J.; Lam, S.; Dietrich, R.A.; Mengiste, T. Expression profiling and mutant analysis reveals complex regulatory networks involved in Arabidopsis response to Botrytis infection. Plant. J. 2006, 48, 28-44. [CrossRef]

20. Liang, Y.; Toth, K.; Cao, Y.; Tanaka, K.; Espinoza, C.; Stacey, G. Lipochitooligosaccharide recognition: An ancient story. New Phytol. 2014, 204, 289-296. [CrossRef]

21. Abdul Malik, N.A.; Kumar, I.S.; Nadarajah, K. Elicitor and Receptor Molecules: Orchestrators of Plant Defense and Immunity. Int. J. Mol. Sci. 2020, 21, 963. [CrossRef]

22. Khoushab, F.; Yamabhai, M. Chitin research revisited. Mar. Drugs 2010, 8, 1988-2012. [CrossRef] [PubMed]

23. Millet, Y.A.; Danna, C.H.; Clay, N.K.; Songnuan, W.; Simon, M.D.; Werck-Reichhart, D.; Ausubel, F.M. Innate immune responses activated in Arabidopsis roots by microbe-associated molecular patterns. Plant. Cell 2010, 22, 973-990. [CrossRef] [PubMed]

24. Xu, G.; Greene, G.H.; Yoo, H.; Liu, L.; Marques, J.; Motley, J.; Dong, X. Global translational reprogramming is a fundamental layer of immune regulation in plants. Nature 2017, 545, 487-490. [CrossRef] 
25. Meteignier, L.V.; El Oirdi, M.; Cohen, M.; Barff, T.; Matteau, D.; Lucier, J.F.; Rodrigue, S.; Jacques, P.E.; Yoshioka, K.; Moffett, P. Translatome analysis of an NB-LRR immune response identifies important contributors to plant immunity in Arabidopsis. J. Exp. Bot. 2017, 68, 2333-2344. [CrossRef] [PubMed]

26. Faus, I.; Ninoles, R.; Kesari, V.; Llabata, P.; Tam, E.; Nebauer, S.G.; Santiago, J.; Hauser, M.T.; Gadea, J. Arabidopsis ILITHYIA protein is necessary for proper chloroplast biogenesis and root development independent of eIF2alpha phosphorylation. J. Plant. Physiol. 2018, 224-225, 173-182. [CrossRef]

27. Lokdarshi, A.; Guan, J.; Urquidi Camacho, R.A.; Cho, S.K.; Morgan, P.W.; Leonard, M.; Shimono, M.; Day, B.; von Arnim, A.G. Light Activates the Translational Regulatory Kinase GCN2 via Reactive Oxygen Species Emanating from the Chloroplast. Plant. Cell 2020, 2, 1161-1178. [CrossRef]

28. Windram, O.; Madhou, P.; McHattie, S.; Hill, C.; Hickman, R.; Cooke, E.; Jenkins, D.J.; Penfold, C.A.; Baxter, L.; Breeze, E.; et al. Arabidopsis defense against Botrytis cinerea: Chronology and regulation deciphered by high-resolution temporal transcriptomic analysis. Plant. Cell 2012, 24, 3530-3557. [CrossRef]

29. Fernández-Bautista, N.; Domínguez-Núñez, J.; Castellano, M.M.; Berrocal-Lobo, M. Plant Tissue Trypan Blue Staining During Phytopathogen Infection. Bio-Protoco 2016, 6. [CrossRef]

30. Berrocal-Lobo, M.; Stone, S.; Yang, X.; Antico, J.; Callis, J.; Ramonell, K.M.; Somerville, S. ATL9, a RING zinc finger protein with E3 ubiquitin ligase activity implicated in chitin- and NADPH oxidase-mediated defense responses. PLoS ONE 2010, 5, e14426. [CrossRef]

31. Abramoff, M.D.; Magalhães, P.J.; Ram, S.J. Image processing with ImageJ. Biophotonics Int. 2004, 11, $36-42$.

32. Toribio, R.; Munoz, A.; Castro-Sanz, A.B.; Merchante, C.; Castellano, M.M. A novel eIF4E-interacting protein that forms non-canonical translation initiation complexes. Nat. Plants 2019, 5, 1283-1296. [CrossRef] [PubMed]

(C) 2020 by the authors. Licensee MDPI, Basel, Switzerland. This article is an open access article distributed under the terms and conditions of the Creative Commons Attribution (CC BY) license (http://creativecommons.org/licenses/by/4.0/). 